\title{
La fidélisation du client par son intégration symbolique dans le discours des entreprises
}

\section{Nathalie Deley}

\section{(2) OpenEdition}

1 Journals

Édition électronique

URL : http://journals.openedition.org/communicationorganisation/3247

DOI : 10.4000/communicationorganisation.3247

ISSN : $1775-3546$

Éditeur

Presses universitaires de Bordeaux

\section{Édition imprimée}

Date de publication : 1 juin 2005

Pagination : 111-123

ISSN : 1168-5549

\section{Référence électronique}

Nathalie Deley, « La fidélisation du client par son intégration symbolique dans le discours des

entreprises », Communication et organisation [En ligne], 27 | 2005, mis en ligne le 07 juin 2012, consulté

le 19 avril 2019. URL : http://journals.openedition.org/communicationorganisation/3247 ; DOI :

10.4000/communicationorganisation.3247

Ce document a été généré automatiquement le 19 avril 2019

(c) Presses universitaires de Bordeaux 


\title{
La fidélisation du client par son intégration symbolique dans le discours des entreprises
}

\author{
Nathalie Deley
}

1 L'article porte sur les magazines de consommateurs, appelés communément par les responsables marketing, les consumer magazines. Leur nombre croissant, estimé à 150 en $2001^{1}$, indique un véritable engouement des entreprises pour cette forme de communication écrite et intéresse de nombreuses enseignes commerciales : les magasins de grande distribution, mais également les magasins spécialisés.

2 Les magazines de consommateurs ne sont pas des dépliants événementiels, édités pour annoncer quelques promotions, mais des rendez-vous réguliers, le plus souvent mensuels ou bimestriels. Ils s'affichent comme de véritables supports de presse adoptant un contenu magazine, moins centré sur les produits et sur les marques et plus ouvert sur les préoccupations des consommateurs. Ils offrent des informations sur la santé, la beauté, l'alimentation, l'éducation des enfants, le sport, mais également sur les orientations de l'enseigne en matière de développement durable, de qualité des produits, de respect de l'environnement... ce qui n'empêche pas certains d'informer en parallèle et dans le même support, leurs lecteurs des réductions et des points de fidélité.

3 Leur diffusion par courrier nominatif ou en libre accès dans les magasins les rend par ailleurs accessibles au plus grand nombre de clients et les positionne comme un des dispositifs de fidélisation les plus prisés actuellement par les enseignes malgré un coût de production et de diffusion élevé. Ils visent en outre à compléter d'autres dispositifs d'intégration, comme les clubs de fidélité, les clubs de testeurs, ou d'autres formules largement promues par le marketing.

4 Nous avons choisi d'étudier ce dispositif en centrant notre analyse sur la figure emblématique du client afin de comprendre les relations que l'entreprise noue avec lui. Pour cela, nous avons relevé les indices de son inscription dans le discours à travers les occurrences textuelles et visuelles d'une part, et les stratégies énonciatives d'autre part. 
5 La problématique s'attache à montrer que la présence du client dans le discours concourt à l'intégrer à l'entreprise. Nous montrerons que les stratégies discursives visent à construire une relation de connivence entre l'enseigne et lui afin qu'il développe un sentiment d'appartenance et lui reste fidèle. Pour cela, nous partirons de l'hypothèse selon laquelle l'entreprise utilise la figure du client dans la construction de son image et accentue la complicité qui les unit en intégrant son discours à l'énoncé journalistique. Le client participe symboliquement au discours commercial et institutionnel de l'entreprise.

6 Dans un premier temps, nous dresserons le portrait du client idéal, tel qu'il est présenté dans les magazines, avec ses différents attributs, et nous définirons deux postures dans l'énonciation de l'acte d'achat. Puis dans une deuxième partie, nous présenterons les modalités d'intervention des clients dans le discours des magazines, notamment en décrivant les témoignages qui sont publiés.

7 Le corpus d'analyse est constitué de gratuits de la grande distribution: «Le journal de Carrefour ", «Vivre Champion », et "Pour vous » de Géant Casino, publiés entre novembre 2004 et février 2005.

\section{La figure emblématique du client}

8 La notion de figure empruntée à la rhétorique depuis Aristote, vient du latin figura " configuration, structure, figure géométrique, manière d'être " et de fingere "façonner, représenter, imaginer ». Utilisée pour désigner dans un premier temps les figures de style littéraire puis pour décrire l'écart entre le signe et le sens (ce qu'on appelle le sens figuré), elle peut par transposition être étendue à l'ensemble du système de signes et être sollicitée par ceux qui s'intéressent à l'étude des représentations. Elle devient dès lors un outil pour décrire la forme que prennent les représentations et renvoie aux stratégies de connotation des énonciateurs. La manière de «parler de » a un sens, une signification qui modifie les représentations des lecteurs.

9 A partir de l'analyse des magazines, il est alors possible de se représenter qui sont les clients idéaux pour l'enseigne, quelles représentations on souhaite donner d'eux et de quelle manière on en parle. Nous avons travaillé sur les images (photographies et dessins) pour repérer les catégories de personnes présentées dans les magazines: 129 représentations ont été comptées et classées. 


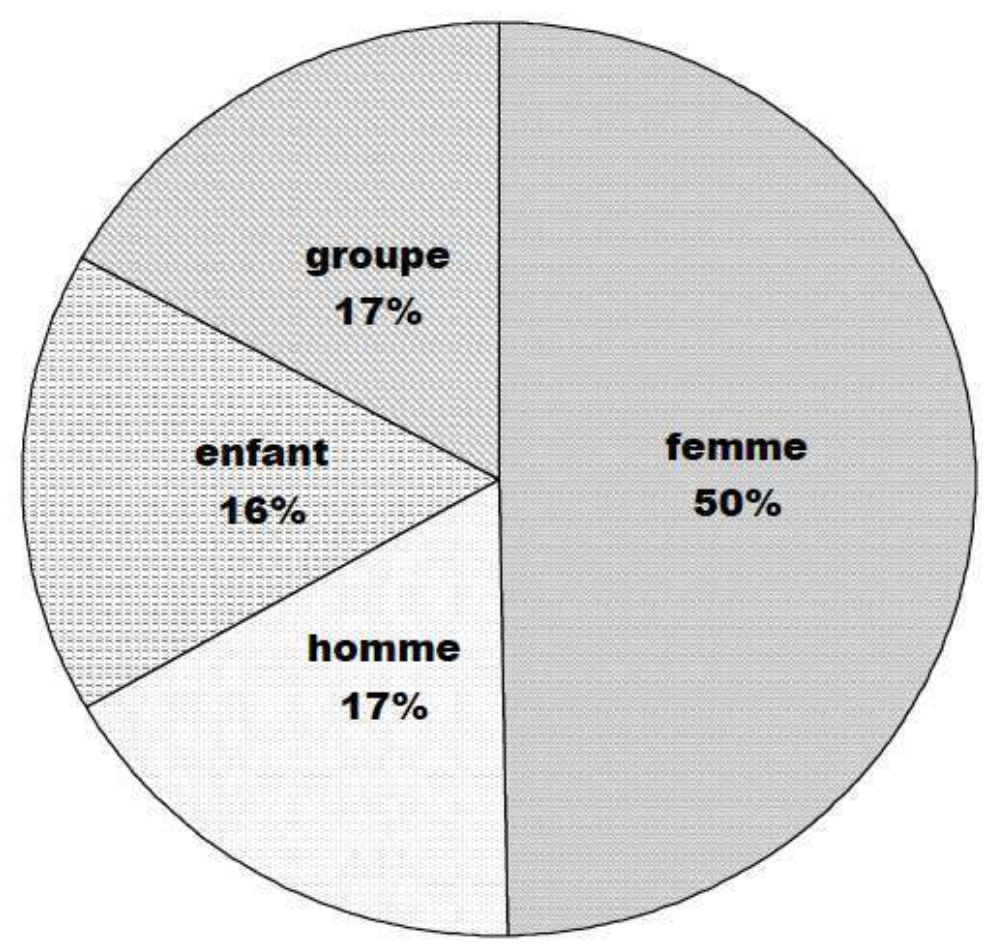

10 Quatre ensembles apparaissent évidents : le premier groupe constitué par des images de femmes est sur-représenté soit 1 image sur 2, auquel on ajoute les $17 \%$ de femmes en groupes accompagnées de leur mari, enfant(s) et ami(s). Le tiers restant représente des hommes et des enfants seuls. La catégorie marketing de la "ménagère de moins de 50 ans » est ainsi confirmée sans aucune surprise.

11 Cependant, il existe des écarts de fréquence entre ces catégories en fonction de l'enseigne éditrice. 
Figure 2

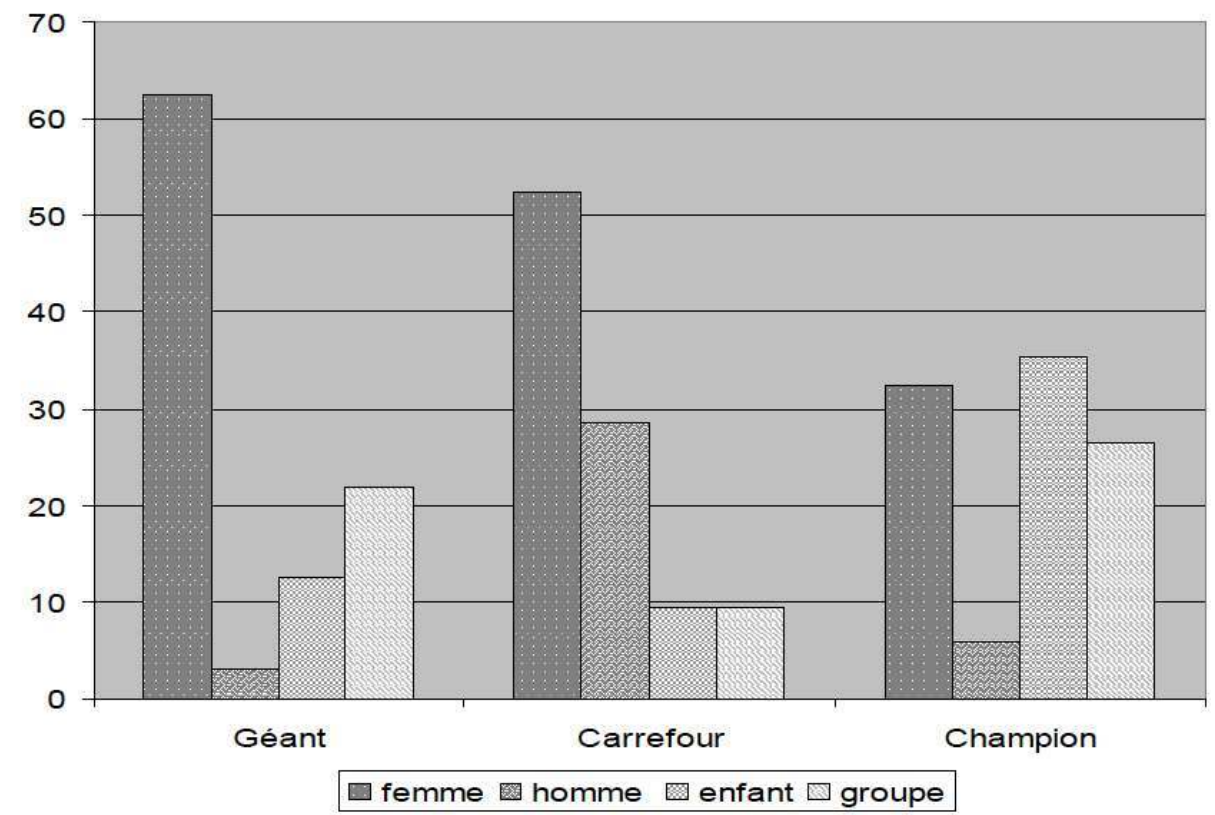
hommes (28,6 \% des images) alors que le journal de Champion met davantage en scène les enfants ( $35,3 \%$ des images contre $16 \%$ sur l'ensemble des titres). «Pour vous » de Géant montre, quant à lui, davantage les femmes : en effet, sur 32 images étudiées une seule représente un homme, ce qui est peu au regard des 62,5\% d'images de femmes.

Pour compléter l'analyse quantitative, nous nous sommes intéressée aux caractéristiques des personnages féminins. Pour cela, nous avons travaillé sur le journal de Géant car il est celui qui, en fréquence, est le plus riche. Dans ce magazine, les femmes ont tout d'abord des âges différents. On remarque ensuite qu'elles ont plusieurs attributs. Elles peuvent apparaître en icônes de mode, à la manière des mannequins en couverture des magazines féminins, mais le plus souvent, elles sont représentées en mère de famille ou en épouse dans un cadre familial chaleureux.

("je suis plus attentive à ce que j'achète »), sur leurs qualités et leurs défauts («je suis trop gourmande »), sur leur situation familiale ("avec mes trois enfants ») et sur leur origine géographique ("originaire de Toulouse, j'adore le cassoulet »). Ces femmes se présentent comme des femmes actives qui n'ont pas le temps de faire des plats traditionnels, des femmes gourmandes, mais au régime, qui soignent leur alimentation, des mères de famille qui pensent à leur entourage, font plaisir à leurs enfants et gèrent le budget familial.

Sont-elles clientes de l'enseigne? Si dans certains cas, la figure du client est clairement identifiée, il arrive fréquemment qu'elle s'efface derrière celle du consommateur: on préfère montrer des personnes qui utilisent les produits de l'enseigne dans leur environnement quotidien plutôt que des acheteurs. L'acte d'achat n'est pas représenté directement. Il découle de la juxtaposition des éléments du discours dans l'espace graphique de la page du journal. 
(1) des personnages fictifs que l'on voit poussant des chariots de supermarché et qui se servent sur les rayons des linéaires- soit en montrant la relation commerciale. Ainsi par exemple sous la rubrique des questions directes, les consommateurs sont toujours des acheteurs : "J'ai acheté une paire de chaussons Tex mais je me suis aperçue plus tard que la taille ne convenait pas.... ${ }^{3}$. Par ailleurs, lorsque les consommateurs témoignent, ils sont photographiés dans les rayons des magasins. On voit très bien en arrière plan des portraits serrés, les linéaires de l'enseigne. La profondeur de champ situe le personnage dans un contexte commercial. Enfin, lorsqu'on rapporte leurs propos, on mentionne toujours " client $(e)$ de ", comme par exemple dans l'article consacré à la clémentine où on peut lire "Comme l'observe Dorothée Uscidda, cliente de Carrefour: "la clémentine cultivée en Corse est donc bien adaptée à son milieu" $"^{4}$.

18 A l'inverse, le magazine « Pour vous » du groupe Géant Casino préfère suggérer plutôt que montrer ou nommer l'acte d'achat. La mise en présence, notamment des témoignages de consommateurs et des descriptions de produits sur l'espace d'une double page, implique que les éléments ne peuvent être interprétés sans référence à leur environnement constitué par d'autres articles, d'autres images, ce que Genette a appelé le texte et le péritexte. En effet, "Quel que soit son degré apparent d'autonomie le message sur lequel l'analyste centre son regard n'est pas, dans l'espace du journal, un élément autonome .... ${ }^{5}$.

19 Par conséquent, la juxtaposition des informations dans l'espace graphique de la page provoque une relation de cause à effet entre l'attente du consommateur, exprimée par le témoin, et la solution offerte par le produit présenté. Les propos de Delphine G.«En m'intéressant à la diététique, j'ai appris à me repérer et à sélectionner les produits qui m'aident à prendre soin de mon équilibre " ${ }^{6}$ sont associés dans un même espace graphique à un bon de fidélité pour des yaourts natures pauvres en cholestérol. L'effet d'association dans la page est une manière de susciter l'acte d'achat.

20 La présentation d'un produit ou d'une gamme de produit, accompagnée d'un coupon destiné à cumuler des points sur la carte de fidélité est une occasion pour encourager le lecteur à consommer et à acheter. Cette présentation s'accompagne souvent de l'avis d'un expert : c'est le plus souvent un homme professionnel, un médecin, un chef de rayon, qui vient confirmer le point de vue de la cliente témoin.

21 La construction narrative de ces témoignages ${ }^{7}$ est toujours la même et reproduit le schéma du texte publicitaire. On met en avant l'existence d'une situation de manque « chaque semaine mes enfants attendent le samedi soir avec impatience ». La prise de conscience de cette situation de manque incite l'énonciateur (l'être) à chercher à la combler et à devenir l'agent d'un faire où apparaît très clairement l'objet de la quête «c'est la soirée croque-vidéo » qui aboutit à une réussite "succès garanti ». L'objet de la quête est toujours présenté comme un objet de valeur positif « avec le cassoulet $R \& R$, c'est facile de se faire plaisir ». Le désir trouve sa concrétisation dans un objet concret, en l'occurrence un produit. Cette stratégie discursive consiste à faire croire qu'il y a un manque qu'on peut combler, grâce à un auxiliaire. 
singulier et le présent de l'énonciation pour rendre compte d'un témoignage particulier. Le «je » ainsi utilisé est interprété par le lecteur grâce à la signature et à la photo d'identité associées au texte. Il rend compte de son expérience sans distinguer le temps de l'énoncé du temps de l'énonciation. Dans le témoignage suivant: «Dans le nouveau rayon, j'apprécie la clarté de la disposition, le choix, les prix ", les deux temporalités coïncident. Le témoignage nous indique que l'énonciateur nous parle de ce qu'il voit. Il n'y a pas de mise à distance, le message n'est pas différé par rapport à la situation vécue. L'allocutaire auquel est destiné ce message doit être capable de déterminer à quoi se réfère cet énoncé. La photo du témoin dans les rayons du magasin, lui permet de situer cet énoncé et de conclure à un témoignage réalisé lors d'une visite par le client dans le rayon du magasin.

Communication et organisation, 27 | 2012 
28 Le «je » du témoignage renvoie à un «tu », il s'adresse à quelqu'un. Le destinataire du message est donc l'allocutaire. Il s'agit la plupart du temps du lecteur, lui-même client de l'enseigne. Cependant, il n'est pas directement interpellé dans le discours direct. L'allocutaire n'apparait pas dans le propos rapporté. Le témoin s'adresse à un allocutaire non déterminé, non inscrit dans le discours. Il n'y a pas de traces de dialogues dans les témoignages. Cependant, le discours est adressé. Et si l'allocutaire n'est pas directement inscrit dans la relation de communication, il apparaît dans le regard de l'énonciateur. En effet, le cadrage serré des portraits des personnes et le regard tourné vers l'objectif signifient que les personnes qui témoignent s'adressent à nous, lecteurs du journal.

Dans « le journal de Carrefour », l'allocutaire est l'enseigne elle-même. Lorsqu'un client demande «Au rayon épices de mon magasin Carrefour, j'ai eu la surprise de ne pas trouver de carvi. Un employé m'a affirmé que le cumin et le carvi, c'était la même chose. Pouvez-vous m'éclairer sur la question? $»^{10}$, le "vous " auquel s'adresse le message, renvoie au médiateur, responsable de la rubrique « questions directes » et représentant de l'enseigne. Il peut s'agir d'un «vous » de politesse ou d'un "vous » collectif, rassemblant les professionnels d'une institution. Les réponses aux questions permettent de valider ce vous collectif car elles sont intitulées "la réponse de carrefour ", elles s'expriment par ailleurs avec un "nous ", qui ne renvoie pas à une multitude d'énonciateurs, mais à un individu qui répond au nom d'une communauté d'intérêt et d'action, car comme le dit D. Maingueneau "Par une sorte de "contrat énonciatif", l'auteur se pose en délégué d'une collectivité investie de l'autorité d'un savoir dont la légitimité repose sur une institution. ${ }^{11}$

30 Par conséquent, la compréhension des actes d'énonciation passe par l'interprétation des éléments contextuels. Il est nécessaire en effet, pour comprendre la portée des énoncés analysés de les mettre en relation avec les autres textes en présence. C'est la combinaison des éléments qui crée l'univers de discours. Ainsi, les témoignages des consommateurs dans le journal de Géant sont appuyés par le texte journalistique, le titre et le questionnement posé, la parole de l'expert encadrée et la présentation d'un produit ou d'une gamme de produits. C'est l'ensemble qui constitue un discours d'implication où le témoin vient dire au destinataire du journal tout l'intérêt qu'il y a à suivre les conseils énoncés. Le discours du témoin est donc un élément argumentatif, mais il n'est pas en soi une argumentation. En effet, le témoin se raconte, plus qu'il n'argumente. L'argumentation naît de la conjonction de tous les éléments. Son discours est donc selon la typologie consacrée, un discours narratif où les actions des témoins et ce qu'ils sont, apparaissent. Ils font les choses, pour eux-mêmes ou pour leur entourage, jamais contre quelqu'un. Leur expérience est positive et leurs propos s'affichent comme une proposition à suivre, à imiter "je me bouge ", " je fais moins d'erreur qu'avant ", "j'apprécie » ${ }^{12}$. Il est rare que le destinataire soit interpellé par le témoin, mais cela peut arriver dans certains cas, qu'on retrouve par exemple dans l'impératif suivant :« essayez, c'est garanti » 13.

31 L'interpellation directe du lecteur intervient en revanche, fréquemment dans l'article journalistique qui accompagne le témoignage ou à travers d'autres dispositifs discursifs comme des encadrés titrés « le saviez-vous? ( journal de Géant). Il arrive également que le «vous» de l'allocution soit présent dans les titres des articles pour conseiller le lecteur: " déclinez vos idées à toutes les sauces». ${ }^{14}$

32 Le témoignage introduit un contrat d'authenticité : les propos renvoient à une réalité, celle de l'énonciateur témoin. Le présent utilisé et la première personne du singulier 
permettent comme le dit $\mathrm{P}$. Charaudeau à propos des titres de presse «de confondre l'instance temporelle de l'activité de lecture et l'instance temporelle de l'événement (ce qui) a pour conséquence de créer (...) l'illusion de direct $»^{15}$. Ainsi, les propos tenus donnent du crédit à l'ensemble des énoncés auxquels ils sont associés. Le témoin se confond avec l'enseigne pour laquelle il témoigne. Il témoigne pour l'enseigne.

Il peut également être sollicité en qualité d'expert. Dès lors, c'est son opinion sur une situation qui est présentée, non son expérience. On utilise ses compétences pour rassembler.

Ainsi, dans la rubrique "A vous de choisir » du journal de Carrefour, deux points de vue contradictoires de consommateurs sont affichés : il y a celui qui est pour et celui qui est contre. Selon les mêmes procédés que ceux évoqués précédemment, les personnes interrogées sont photographiées, et identifiées par leur nom, leur âge et leur profession. Ces experts sont investis d'un savoir dont ils rendent compte en argumentant. Ils accèdent de cette manière au statut de conseiller. Ce ne sont cependant pas des personnes quelconques, le métier qu'elles exercent les autorise à avoir un avis, mais ce ne sont pas des professionnels de l'enseigne. On pourrait penser que grâce à eux le lecteur va faire son choix librement, mais en fait, le journal attend de lui un comportement qui va être induit par l'énoncé principal et par le face à face des arguments des deux locuteurs.

L'analyse d'un exemple peut nous éclairer. Il s'agit de présenter une nouvelle forme de publicité commerciale sur téléphone portable: «La pub par SMS $»^{16}$. Trois énoncés constituent la rubrique : un texte journalistique, et deux points de vue contradictoires. L'énoncé journalistique précise le cadre de mise en application des messages publicitaires sur les téléphones portables : "Ces messages sont courts... concis, informatifs - annonce d'une promotion, arrivée d'un nouveau modèle chez un concessionnaire auto... la législation est drastique: pour recevoir ces SMS, il faut avoir donné expressément son accord -résiliable à tout moment-... les fichiers sont secrets ». L'article ne fait pas référence à l'enseigne Carrefour. Deux énonciateurs experts donnent leur position. Le premier dans l'ordre de la page est "pour»: "cela te permet d'être prévenu d'événements sans être vraiment dérangé. Et c'est rapide... Mais c'est plutôt un truc de jeunes... Si Carrefour me proposait une offre commerciale par SMS à mon arrivée au magasin, cela m'intéresserait, j'aurais l'impression d'être un peu privilégié ", C.M. 20 ans, commerciale. Le deuxième y est opposé, il précise que cela peut être " agaçant... vos coordonnées peuvent se retrouver chez n'importe qui. " A.B., 60 ans expublicitaire.

On constate que les arguments avancés trouvent des réponses dans l'article principal et dans l'articulation des énoncés entre eux. Par ailleurs, la position des énoncés dans la page induit une orientation du lecteur, car comme le disent M. Mouillaud et J.F. Tétu « S'il est vrai... que la citation n'existe que dans la mise en scène de l'écriture (notamment par la présence des guillemets ou d'un choix typographique différent de l'énoncé principal), le journal, en tant qu'écriture de l'espace, dispose d'une scène spécifique, une scène où se jouent des opérations stratégiques... $»^{17}$

37 Les citations entendues comme discours direct enchâssé dans un énoncé principal sont rares dans les consumer magazines. Elles supposent une articulation de deux discours où le plus souvent le discours rapporté de la citation est encadré par un discours plus fort, soutenu par le journal. Pour que la citation ne s'efface pas derrière l'énoncé qui le cite, il est nécessaire que le discours journalistique reconnaisse une légitimité et une valeur aux propos rapportés. Le journal est alors, comme l'écrivent M. Mouillaud et J.F. Tétu « une "chambre d'échos", il est tributaire des voix qu'il reproduit....1 ${ }^{18}$, les énonciateurs sont renvoyés 
face à face avec leur réalité propre : celle de l'énoncé journalistique et celle de la citation. La citation sert donc à asseoir cette confrontation qui, dans le consumer magazine, a peu de raison d'être. Il y a peu de place, en effet, pour des réalités différentes.

Par conséquent, dans le corpus étudié, seul «le journal de Carrefour »utilise ce dispositif discursif. Dans les dossiers consacrés à la présentation d'un produit, des clients sont invités par l'enseigne à aller sur les lieux de production ou dans les services de recherche et développement et à rendre compte de ce qu'ils ont pu observer lors de leur visite : « "La sécurité est vraiment maximum", s'exclame, visiblement impressionnée, Ayse Canli, cliente Carrefour. $"{ }^{19} \mathrm{Il}$ n'y a pas de risque de discordance entre les deux niveaux d'énonciation, celui du consommateur, celui de l'enseigne. Les discours cités viennent renforcer le discours premier. Aucune mise à distance n'est nécessaire. Le discours rapporté est au service de l'énoncé journalistique.

\section{En conclusion}

Les consumer magazines sont sans aucun doute des supports marketing même si les enseignes cherchent à les afficher comme de véritables médias d'information. Certaines ont opté pour une diffusion payante. «Epok» de la FNAC, "Maisons en vie » de LEROY MERLIN et «Room » d'IKEA ${ }^{20}$ jouent la carte du titre de presse. Mais, il ne faut pas se méprendre, ces titres restent au service de la stratégie de l'entreprise. L'indépendance journalistique ne semble pas envisageable et le commanditaire reste déterminant dans le choix des informations et dans la manière de les présenter. Les lecteurs doivent savoir immédiatement à quelle marque appartient un titre. Les consumer magazines interviennent dans le marketing relationnel entre l'enseigne et les clients, au même titre qu'un programme de fidélité.

Par conséquent, les magazines doivent répondre à trois impératifs: servir l'image de l'entreprise, faciliter sa relation avec le client et intéresser le public lecteur.

41 L'analyse que nous avons menée sur trois titres de la grande distribution, nous a tout d'abord montré que le client représenté était essentiellement féminin. Cette figure et ses caractéristiques (âge, qualité, compétence) sont le résultat des connaissances que les enseignes ont de leurs clients. A partir d'analyses statistiques complexes élaborées sur les données recueillies à chaque passage en caisse grâce aux cartes de fidélité ou aux cartes de paiement, les enseignes élaborent en effet, des profils types de clients. Elles connaissent ainsi la date de leur dernière visite, le nombre de passages mensuels dans l'enseigne et le montant moyen de leur panier. Couplées avec les informations sur l'identité du possesseur (âge, profession, adresse, revenu, centres d'intérêt) obtenues à l'ouverture de la carte, elles ont une représentation assez précise des habitudes et des caractéristiques de consommation de leurs clients.

L'analyse nous a ensuite permis de voir que le lecteur des consumer magazines se confondait avec le client, et que ce dernier tantôt énonciateur ou allocutaire du discours énoncé dans ces supports, participait à la valorisation de l'enseigne émettrice, en légitimant son propre discours et en validant l'acte d'achat, qui demeure le principal enjeu de ce type de support. Le consommateur peut ainsi se reconnaitre dans le magazine et adhérer aux propositions, conseils, suggestions qui lui sont faites, dans la mesure où il est lui-même sollicité pour leur élaboration à travers son expérience et son témoignage. 
43 contenu des magazines renforce l'identification des lecteurs et répond aux trois impératifs énoncés ci-dessus. L'image de l'entreprise est valorisée car elle se présente soucieuse de ses clients, sa relation avec eux est renforcée et le public lecteur peut plus facilement s'intéresser aux informations données puisqu'elles apparaissent avec des visages familiers.

Cependant, le lecteur peut se questionner sur l'existence réelle de ces personnes et sur la validité des témoignages. Un doute subsiste tant que ces titres restent dépendants des marques pour lesquelles ils sont édités. C'est la raison pour laquelle ils cherchent sans cesse à renforcer la confiance par différentes stratégies. Ainsi, le magazine « Pour Vous » de Géant fait participer le lecteur au contenu rédactionnel du journal en lui demandant des astuces pour améliorer le quotidien. La meilleure astuce du mois publiée dans le journal, est ensuite récompensée par des points de fidélité.

\section{BIBLIOGRAPHIE}

Bardin L., L'analyse de contenu, PUF, 1977.

Bautier R., De la rhétorique à la communication, PUF, 1994.

Beslu F.X., « Ceux qui vous mettent en fiches », L'express, 13 juin 2002.

Cristofari J.F., « Ecrits de marque », Marketing magazine, juin août 2001.

Charaudeau P., Langage et discours, Hachette, 1988.

Genette G., Les figures du discours, Flamarion, 1968.

Mouillaud M. et Tétu J.F., Le journal quotidien, Presses Universitaires de Lyon, 1989.

Maingueneau D., L'énonciation en linguistique française, Hachette, 1999.

Lavoigne Y., Le langage des médias, PUG, 1997.

\section{NOTES}

1. D'après l'étude menée par J.M. Kehr et publiée en novembre 2001, agence Eurostaff

2. publiés dans « Pour Vous» de nov. 2004 à janv. 2005

3. publiés dans « Le journal de Carrefour » $\mathrm{n}^{\circ} 12$, fév. 2005

4. idem $\mathrm{n}^{\circ} 10$, déc. 2004

5. Y. Lavoine, 1997

6. «Pour vous » nov. 2004

7. extraits de « Pour vous » janv. 2005

8. D. Maingueneau, 1999

9. «Le journal de Carrefour » $\mathrm{n}^{\circ} 10$, déc. 2004

10. Ibid., n 11, janv. 2005

11. D. Maingueneau, 1999, p. 32

Communication et organisation, 27 | 2012 
12. «Pour vous » de nov. 2004 à fév. 2005

13. «Pour vous", nov. 2004

14. Ibid.

15. P. Charaudeau, 1988

16. «Le journal de Carrefour » $n^{\circ} 11$, janv. 2005

17. M. Mouillaud et J.F. Tétu, 1989, p. 135

18. Ibid., p. 134

19. «Le journal de Carrefour » $n^{\circ} 11$, janv. 2005

20. Cette stratégie ne concerne que $11 \%$ des titres.

\section{RÉSUMÉS}

Cet article apporte un éclairage sur les stratégies de fidélisation des entreprises à partir de l'analyse des consumer magazines qu'on appelle également selon les cas, presse de consommateurs ou magazine de marque. Il s'agit de montrer la place des co-énonciateurs dans le discours de trois titres, en insistant plus spécialement sur la figure du client. Ce dernier apparaît en consommateur expérimenté qui désigne par son témoignage, l'acte d'achat qui le lie à l'entreprise. Il participe à la définition que l'enseigne souhaite donner de son image et renforce de cette manière leur connivence. En intervenant au côté des énoncés journalistiques, il porte le discours commercial de l'enseigne.

This article analyses the strategy of fidelisation of consumer magazines. We show the strategy of enonciation through the analysis of three titles : «Le journal de Carrefour ", "Vivre champion » and «Pour Vous » by Géant Casino. We study mainly the customers' testimonies in short articles. These quotations show the relation between buyers and retailers. They are the symbols of the partnership existing between sales outlets and their customers.

\section{AUTEUR}

\section{NATHALIE DELEY}

Nathalie Deley est Maître de Conférences à l'Université Pierre Mendès France (Grenoble 2). Chercheur à Lyon 2, équipe média et identité, son domaine de recherche est l'analyse des supports de presse professionnelle (presse d'entreprise, presse syndicale, presse consulaire, consumer magazines). Mail : Nathalie.deley@wanadoo.fr 